\section{REGULATORY T CELLS}

\section{Mst kinase roles}

Immunity https://doi.org/10.1016/

j.immuni.2018.10.010 (2018)

Regulatory $\mathrm{T}$ cells ( $\mathrm{T}_{\mathrm{reg}}$ cells) require the cytokine IL-2 for their suppressive activity, yet $\mathrm{T}_{\text {reg }}$ cells are adapted to low concentrations of IL-2, which has prompted questions about how efficient use of IL-2 is achieved. In Immunity, Chi and colleagues show that $\mathrm{T}_{\text {reg }}$ cells utilize the Hippo signal-transduction pathway to amplify IL-2-mediated activation of the transcription factor STAT5. Kinaseinhibitor screens identify roles for the Hippo kinases Mst1 and Mst2 in $\mathrm{T}_{\text {reg }}$ cells. Mice with $\mathrm{T}_{\text {reg }}$ cell-specific loss of Mst1-Mst2 exhibit a scurfy-like autoimmune phenotype and die prematurely. Loss of Mst kinases impairs $\mathrm{T}_{\text {reg }}$ cell lineage stability and survival in response to IL-2. Mechanistically, Mst1 interacts with the actin cytoskeleton regulators DOCK8, $\mathrm{LRCH}$ and Rac1, which regulate T cell mobility. Consistent with that finding, Mst kinase-deficient $\mathrm{T}_{\text {reg }}$ cells display defective homing and positioning within lymphoid organs, in addition to defective activation of phosphorylated STAT5 that compromises their suppressor function.

https://doi.org/10.1038/s41590-018-0284-y

\section{GENE THERAPY}

\section{Cas9-directed immunity}

Nat. Med. https://doi.org/10.1038/s41591-018-

0204-6 (2018)

The CRISPR-Cas9 nuclease system holds great promise for therapeutic manipulation of the genome, but neutralizing immunity to this bacteriaderived product could severely limit its utility in vivo. In Nature Medicine, Schmueck-Henneresse and colleagues investigate whether the normal human population has pre-existing $\mathrm{T}$ cell immunity to the Cas9 component derived from Streptococcus pyogenes (SpCas9), a bacterium that causes common childhood infections. Both CD4 ${ }^{+}$effector $\mathrm{T}$ cells and $\mathrm{CD}^{+}$effector $\mathrm{T}$ cells respond to Cas9 in almost all donors assessed. In addition, responses to SpCas9 orthologs derived from Acidaminococcus and Staphylococcus are detected. Regulatory T cells also respond to SpCas9, which suggests that although pre-existing T cell immunity to Cas 9 is prevalent in the human population, it might be mitigated by regulatory T cell-mediated suppression.

https://doi.org/10.1038/s41590-018-0285-x

\section{NEUROIMMUNOLOGY}

\section{Blood-brain barrier integrity}

Proc. Natl. Acad. Sci. USA https://doi.org/10.1073/ pnas.1808609115 (2018)

Aging is associated with progressive systemic increases in inflammation that can contribute to neurodegeneration. In the Proceedings of the National Academy of Sciences, Mucke and colleagues use a mouse model to investigate the choroid plexus (CP), a major gateway that regulates entry of cells of the immune

\section{ANTIGEN PRESENTATION} \section{CDC1 cross-priming}

system into the brain. They observe that expression of the anti-aging protein Klotho decreases in the CP of aged mice. Experimental depletion of Klotho specifically in the CP results in increased inflammatory signatures in the CP, infiltration by macrophages and enhanced microglial activation in the brain in response to lipopolysaccharide challenge. In vitro modeling shows that Klotho regulates inflammation by cooperating with signaling via the fibroblast growth factor FGF23 to reduce activation of the NLRP3 inflammasome. High levels of Klotho in the CP might therefore enhance blood-brain barrier integrity.

https://doi.org/10.1038/s41590-018-0286-9

\section{GUT EPITHELIUM \\ Stem cell-immune cell cross-talk \\ Cell https://doi.org/10.1016/j.cell.2018.10.008 (2018)}

The gut epithelium constantly regenerates through the rapid proliferation and differentiation of intestinal stem cells (ISCs). In Cell, Biton et al. identify two subpopulations of proliferative Lgr5 ${ }^{+}$ISCs that express major histocompatibility class II (MHCII), process extracellular antigens and activate naive $\mathrm{T}$ cells. In intestinal organoid cultures, the addition of regulatory $\mathrm{T}$ cells or the anti-inflammatory cytokine IL-10 leads to the expansion of ISC populations; addition of the $\mathrm{T}_{\mathrm{H}} 1$ subset of helper $\mathrm{T}$ cells or the cytokine IFN- $\gamma$ induces the differentiation of ISCs into Paneth cells; and the addition of $\mathrm{T}_{\mathrm{H}} 2$ cells or the cytokine IL-13 induces the differentiation of ISCs into tuft cells. In mice, infection with Salmonella enterica or Heligmosomoides polygyrus increases the frequency of $\mathrm{MHCII}^{+}$ ISCs and the number of Paneth cells or tuft cells, respectively. Mice that lack expression of MHCII on Lgr5 $5^{+}$ISCs or $\mathrm{T}$ cell-deficient mice have an increased number of ISCs. Regulatory T cell-deficient mice have a lower frequency of quiescent $\mathrm{Lgr}^{+}$ISCs, an increased frequency of $\mathrm{MHCII}^{+}$ISCs and increased proliferation and differentiation of ISCs into Paneth and tuft cells.

https://doi.org/10.1038/s41590-018-0282-0

Laurie A. Dempsey, Zoltan Fehervari and loana Visan 